\title{
MANNOSE-SPECIFIC IMMUNOGLOBULIN M IN THE MUCUS OF FUGU TAKIFUGU RUBRIPES IS UTILIZED BY A MONOGENEAN PARASITE HETEROBOTHRIUM OKAMOTOI FOR HOST RECOGNITION
}

\author{
Shigeyuki Tsutsui ${ }^{1}$, Kento Igarashi ${ }^{1}$, Sachi Hirakawa ${ }^{2}$, Sho Hosoya ${ }^{3}$, \\ Hiroaki Suetake ${ }^{2}$, Kiyoshi Kikuchi ${ }^{3}$, Yuzuru Suzuki ${ }^{3}$, Osamu Nakamura ${ }^{1}$, \\ Satoshi Tasumi ${ }^{3}$, Toshiaki Miyadai ${ }^{2}$ \\ ${ }^{1}$ School of Marine Biosciences, Kitasato University, Japan \\ ${ }^{2}$ Faculty of Marine Bioscience, Fukui Prefectural University, Japan \\ ${ }^{3}$ Fisheries Laboratory, University of Tokyo, Japan
}

\begin{abstract}
How parasites recognize their definitive hosts is a mystery; however, it is likely that parasitism is initiated through the molecular recognition of certain molecules on host surfaces by parasites. Fish ectoparasites make the initial contact with their hosts at the body surface, including the skin and gills, which is covered with a mucous layer. Fish body mucus contains several types of molecules acting as defense factors. The present study provides new evidence demonstrating that mannose-specific IgM in the gill mucus is utilized by a monogenean parasite, Heterobothrium okamotoi, to recognize their host, fugu (Takifugu rubripes). First, we determined that the infective parasite larvae, the oncomiracidium, shed their cilia and metamorphosed into juveniles when exposed to mannose-binding proteins partially purified from fugu skin or skin mucus. These proteins were identified as mannose-specific IgM and two mannose-binding lectins, pufflectin and kalliklectin. Among these, deciliation was significantly induced by IgM and inhibited by mannose or the specific antibody against fugu IgM, whereas the other proteins had no effect. Immunofluorescent staining revealed that fugu mannose-specific IgM bound to the ciliated epithelial cells of the oncomiracidium. In contrast, IgM without mannose affinity induced deciliation at significantly lower levels. These findings suggest that the binding of fugu $\operatorname{IgM}$ to a ligand present on the cell surface via the antigen-binding site triggered deciliation of oncomiracidium. The mannose-binding IgM concentration in the gill mucus where the parasite colonized was high enough to induce deciliation in vitro, indicating that $H$. okamotoi initially uses the defense molecule for establishing parasitism.
\end{abstract}

\section{KEYWORDS}

Fugu, Heterobothrium okamotoi, Host recognition, IgM, Mannose, Parasite, 